\title{
Parallel Files Distribution
}

\author{
Laurentiu Cucos ${ }^{1}$ and Elise de Doncker ${ }^{1}$ \\ Western Michigan University \\ \{lcucos, elise\}@cs.wmich.edu \\ http://aegis.cs.wmich.edu/ 1 cucos \\ http://www.cs.wmich.edu/ ${ }^{\text {elise }}$
}

\begin{abstract}
For parallel computations requiring massive data Input/Output, one of the goals is to maximize the use of the underling storage topology, in particular exploit the benefit of using local disks. This paper presents a mechanism to distribute independent data records from multiple files to multiple processing nodes and vice-versa. An allocation problem is solved using the Max-Flow algorithm. We give timing results using various read/distribute protocols. One application is to redistributing tasks (regions) for restarting parallel numerical integration runs.
\end{abstract}

\section{Introduction and Motivation}

In order to maintain a minimum transport cost between producers, consumers and warehouses, it is preferable to have all three within the same system, in close proximity, or use paths of easy access between one another. In addition, is desired to have a small number of moves or stops between producers and consumers.

As in an industrial scenario, parallel computations that produce, store and later consume large amounts of data, have to follow similar principles.

Let us consider an arbitrary topology of high speed connections between a number of organizations, each providing a number of machines with either local storage and/or access to shared partitions. The optimum distribution would be achieved when the data is generated, saved, and loaded in the same computing node (keeping all other data transfers at a minimum); however, this may not always be possible or efficient. For irregular problems, some nodes may generate more data than they can store, reload and re-process, or just more data than other nodes. Under these conditions it would be difficult to predict and control how the data should be distributed.

Addressing mainly scenarios where data need to be read only once, this paper presents an efficient distribution engine.

As an application we consider redistributing regions in between calls to numerical multivariate integration codes. PARINT (Parallel Integration project) $[1$ 5. implements adaptive partitioning (as well as other) strategies for multivariate integration.

Section 2 below outlines a general equal-distribution algorithm, Section 3 gives experimental results and Section 4 concludes the paper. 


\section{$2 \quad$ Static Distribution}

To avoid synchronization overheads, and since the total amount of work is known, it is more efficient to compute the data distribution in advance. While in most cases data can be easily preallocated by distributing equal shares among all machines, non-trivial interconnection networks require more sophistication.

Consider the following general allocation problem [4]:

Problem: Given a set of $n$ machines, $\mathrm{M}=\left\{m_{i}\right\}_{1 \leq i \leq n}$, a collection of $p$ files, $S=\left\{s_{j}\right\}_{1 \leq j \leq p}$, where $s_{j}$ has $\left|s_{j}\right|$ records, and a unidirectional bipartite graph $G=\{(M, S), E\}$, where $E=\left\{\left(v_{i}, u_{j}\right)\right\}$ with $v_{i} \in M$ and $u_{j} \in S$, which defines a mapping between machines and files, compute the item distribution so that each machine gets an equal share of records. In case that an equal distribution cannot be achieved, find a solution with records shipped between machines at a minimum communication cost.

Example: Four computing nodes $m_{1}, m_{2}, m_{3}, m_{4}$, participating in a computation requiring data from three file servers $F S_{1}, F S_{2}, F S_{3}$; where $m_{1}, m_{2}, m_{4}$ can access $F S_{1}, m_{1}, m_{3}$ can access $F S_{2}$, and all can access $F S_{3}$. All machines are interconnected by the same type of network.

Solution: An efficient distribution can be achieved using a Min-Cut Max-Flow algorithm [3] and the following procedure.

1. Augment the graph with two new nodes: source and sink, and edges between all set-nodes and source, and all machine-nodes and sink.

2. Assign infinite capacities to edges between sets and machines, $\left|S_{i}\right|$ between set $S_{i}$ and source, and $\left\lceil\frac{\sum_{i=1}^{p}\left|S_{i}\right|}{n}\right\rceil$ between each machine and the sink.

3. Apply the Max-Flow algorithm to the newly created graph.

4. If the resulting flow is $\sum_{i=1}^{p}\left|S_{i}\right|$, there exists an equal distribution of the sets; the distribution is given by the flow in the inner edges. Otherwise, the sets cannot be equally distributed without additional communication and go to step 5 .

5. Equally distribute the records not allocated in step 3 to machines with a smaller number of records.

\section{$3 \quad$ Experiments}

We implemented the system PFD which is a parallel read/write engine to distribute data records from/to files located over multiple machines. It provides a transparent and efficient way to incorporate data reading, distributing, and processing in the same program. The system is layered over the MPI message passing library [2].

PFD was mainly developed for ParInt, to save/load large numbers of integration regions; however it can be easily integrated in any application that reads/writes large numbers of independent records. 
Given a collection of records distributed over a number of machines, PFD:

- reads each record exactly once (when the record is processed);

- minimizes the communication and efficiently distributes all records among all participating nodes;

- provides an easy and transparent way to use local disks;

- uses a buffer of configurable size for interprocess communication;

- provides an array of options to read and distribute the data such that:

- each node will process the same number of records (EQUAL),

- or allow faster nodes to process more data (COMPETE),

- or, only nodes with direct access to data will process records (LOCAL).

The system is written in $\mathrm{C}$, and can be integrated with the user application trough a small set of read/write/pack/unpack functions.

We performed a set of experiments reading $1 \mathrm{~GB}$ of data organized in $8 \mathrm{~KB}$ records and using different distributions, ranging from all data being stored in
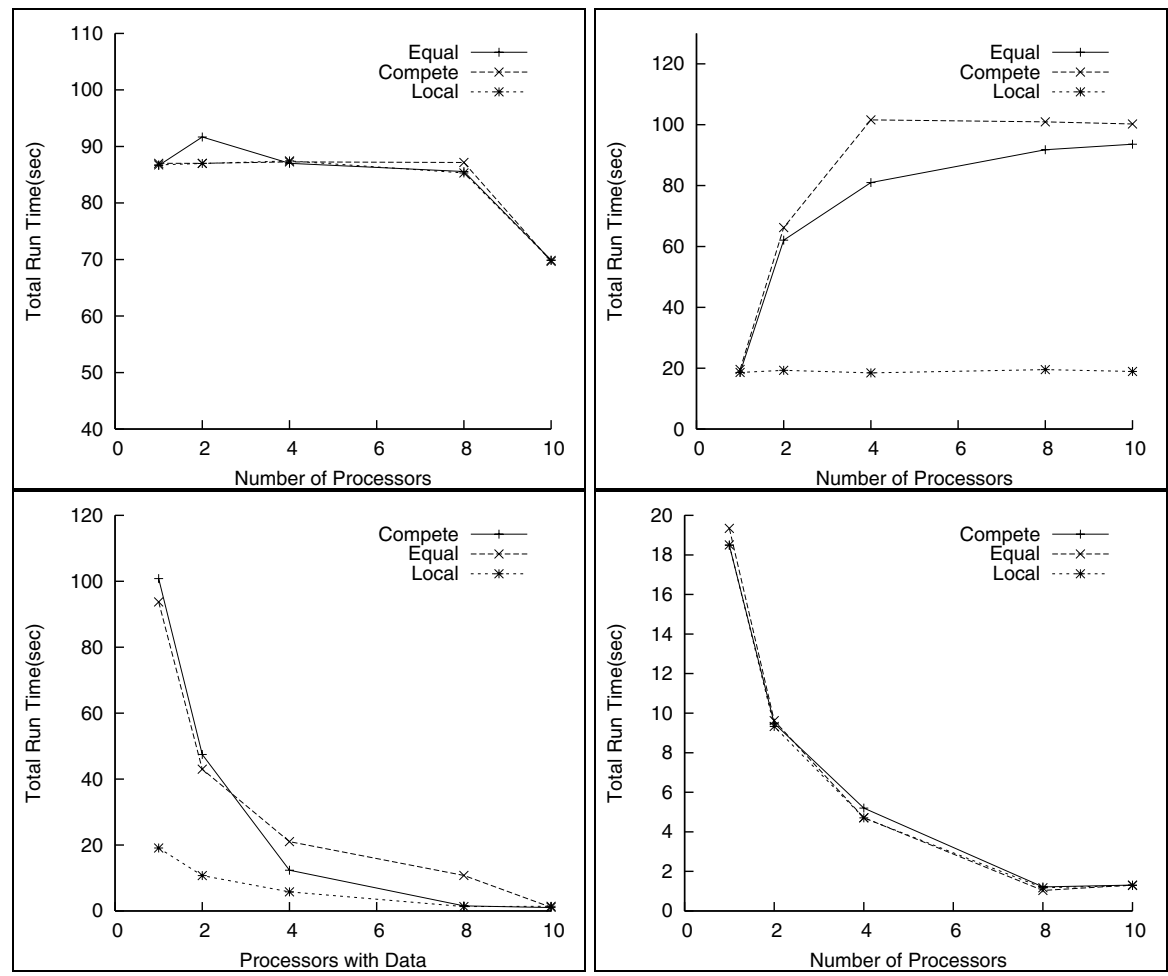

Fig. 1. Top-Left: Distribute records from a shared partition; Top-Right: Distribute records from one non-shared partition; Bottom-Left: Distribute records located in a subgroup of machines; Bottom-Right: Distribute records shared equally by the participants 
one shared partition, to all data located in one local disk. Up to 10 readers are selected from a set of $1.2 \mathrm{GHz}$ AMD Athlon processor nodes, communicating on a Fast Ethernet network.

Figure 1 (Top-Left) shows results for data located in a shared partition. In this case, the runtime is independent of the read method (EQUAL, CompeTE, or LOCAL), and of the number of processors, and is mainly driven by the network transfer speed. Note that the shared partition of this experiment was located on a disk significantly faster than the local disks used in the following experiments.

Figure 1 (Top-Right) shows results for data located in one non-shared partition. While the total read time is around $20 \mathrm{sec}$, the performance quickly depreciates as more processors are involved in the computation. We attribute this behavior to the bottleneck at the distribution node.

Figure1(Bottom-Left) and (Bottom-Right) show results for data non-uniformly distributed, and uniformly distributed, respectively, over participating machines. There is a significant improvement in runtime and scalability. In the latter case, the read time was not affected by the distribution method (Equal, Compete, or Local).

\section{Conclusions}

This paper outlines a mechanism to distribute independent data records from multiple files to multiple processing nodes and vice-versa. We present a solution to the general allocation problem of sharing the data equally among the participating compute nodes, given an arbitrary storage topology.

Although using local disks normally binds data producers with consumers, our system maintains the benefit offered by this connection, while giving at the same time a set of options to share data with non-producers.

\section{Acknowledgments}

This work is supported in part by Western Michigan University, and by the National Science Foundation under grants ACI-0000442, ACI-0203776, EIA0130857.

\section{References}

1. http://www.cs.wmich.edu/parint, PARINT web site.

2. http://www-unix.mcs.anl.gov/mpi/index.html, MPI web site.

3. Cormen, T. H., Leiserson, C. E., And Rivest, R. L. Introduction to Algorithms. The MIT press, 1994.

4. Cucos, L. Load Sharing Strategies in Distributed Environments. PhD thesis, Western Michigan University, December 2003.

5. Zanny, R., De Doncker, E., Kaugars, K., And Cucos, L. ParInt1.2 User's Manual. Available at http: //www.cs.wmich.edu/parint. 